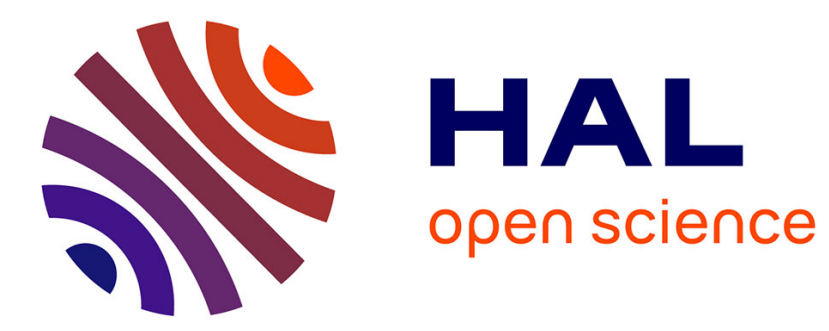

\title{
Simultaneous sparsity-based binary hypothesis model for real hyperspectral target detection
}

\author{
Ahmad Bitar, Loong-Fah Cheong, Jean-Philippe Ovarlez
}

\section{To cite this version:}

Ahmad Bitar, Loong-Fah Cheong, Jean-Philippe Ovarlez. Simultaneous sparsity-based binary hypothesis model for real hyperspectral target detection. 2017 IEEE International Conference on Acoustics, Speech and Signal Processing (ICASSP 2017), Mar 2017, New Orleans, United States. 10.1109/ICASSP.2017.7953031 . hal-01692408

\section{HAL Id: hal-01692408 \\ https://hal-centralesupelec.archives-ouvertes.fr/hal-01692408}

Submitted on 27 Mar 2020

HAL is a multi-disciplinary open access archive for the deposit and dissemination of scientific research documents, whether they are published or not. The documents may come from teaching and research institutions in France or abroad, or from public or private research centers.
L'archive ouverte pluridisciplinaire HAL, est destinée au dépôt et à la diffusion de documents scientifiques de niveau recherche, publiés ou non, émanant des établissements d'enseignement et de recherche français ou étrangers, des laboratoires publics ou privés. 


\title{
SIMULTANEOUS SPARSITY-BASED BINARY HYPOTHESIS MODEL FOR REAL HYPERSPECTRAL TARGET DETECTION
}

\author{
Ahmad W. Bitar ${ }^{1}$, Loong-Fah Cheong ${ }^{2}$ and Jean-Philippe Ovarlez ${ }^{1,3}$ \\ ${ }^{1}$ SONDRA/CentraleSupélec, Plateau du Moulon, 3 rue Joliot-Curie, F-91190 Gif-sur-Yvette, France \\ ${ }^{2}$ National University of Singapore, Singapore, Singapore \\ ${ }^{3}$ ONERA, DEMR/TSI, Chemin de la Hunière, 91120 Palaiseau, France
}

\begin{abstract}
In this paper, a simultaneous sparsity representation-based binary hypothesis (S-SRBBH) model for target detection in hyperspectral image (HSI) is proposed. The S-SRBBH exploits the interpixel correlation within neighboring pixels in HSI, and then, each test pixel is represented by only the background dictionary $\left(\mathbf{A}_{b}\right)$ under null hypothesis or from the union of $\mathbf{A}_{b}$ and target dictionary $\left(\mathbf{A}_{t}\right)$ under alternative hypothesis. Usually, an inner window region (IWR) centered within an outer window region (OWR) contribute in constructing $\mathbf{A}_{b}$. Indeed, the use of IWR has a huge effect on the detection performance since it encloses the targets of interests, but its use requires the information of the size of the targets which is usually hardly available. That is why, this paper also serves to construct $A_{b}$ without IWR by exploiting the low-rank and sparse matrix decomposition (LRaSMD) technique to decompose the HSI into low-rank background HSI and sparse target HSI. Then for each test pixel, a concentric window is located on the low-rank background HSI, and all the pixels within the window contribute to form $\mathbf{A}_{b}$. Two real HSIs are used to demonstrate that S-SRBBH achieves good target detection especially when the LRaSMD technique is exploited to construct $\mathbf{A}_{b}$.
\end{abstract}

Index Terms - Hyperspectral image, simultaneous sparsity, binary hypothesis, inner window, low-rank

\section{INTRODUCTION}

Hyperspectral image (HSI) consists of simultaneously acquiring the same spatial scene in a very narrow and contiguous spectral wavelength (color) bands [1, 2]. Hence, the resulting HSI is a 3-D data cube which provides both spatial and spectral information. With the rich information afforded by the high spectral dimensionality, target detection is not surprisingly one of the most important applications of HSI, where each pixel $\mathbf{x} \in \Re^{p}$ is labeled as target or background based on their spectral signatures [1, 2, 3, 4, 5, 6, 7]. Usually, the detection is built using a binary hypothesis test that chooses between the following competing null and alternative hypothesis: target absent $\left(H_{0}\right)$, that is, the test pixel, $\mathbf{x}$, consists only of background; and target present $\left(H_{1}\right)$ where $\mathbf{x}$ may be either fully or partially occupied by the target material. A lot of algorithms proposed for hyperspectral target detection use only a single spectrum for the detection. Well known algorithms under this category include the following, with different binary hypothesis models: adaptive matched filter (AMF) [8. 9], adaptive normalized matched filter (ANMF) [10], adaptive Kelly detector [11].

In fact, using only a single spectrum for the detection is usually insufficient to achieve good target detection since the spectrum changes with the environmental situation. A lot of algorithms have been developed to take the variability into account. Well known algorithms include: matched subspace detector (MSD) [12], adaptive subspace detector (ASD) [13].

The main drawback of all the classical detectors cited above is that there is always an explicit assumption (Gaussian) on the statistical distribution characteristics of the observed data. To do away with such assumption, Chen et al. [14] developed a target detection algorithm based on sparse representation for HSI data. In particular, each test pixel in HSI, be it target or background, is assumed to lie in a low dimensional subspace and thus can be represented by a very few training samples, including both $\mathbf{A}_{b}$ and $\mathbf{A}_{t}$. Zhang et al. [15] proposed the sparsity representation based binary hypothesis (SRBBH) model in which they combined the idea of binary hypothesis and sparse representation to obtain a more complete and realistic model than in [14]. More precisely, if the test pixel belongs to $H_{0}$, it will be modeled by the $\mathbf{A}_{b}$ only; otherwise, it will be modeled by the union of $\mathbf{A}_{b}$ and $\mathbf{A}_{t}$. This in fact yields a competition between the two hypothesis corresponding to the different pixel class label.

The SRBBH model which has been developed very recently does not take into account any joint sparsity model that can incorporate the interpixel correlation within the HSI by assuming that neighboring pixels usually consist of similar materials and thus have the same spectral characteristics [16]. In addition, in both [14] and [15], the $\mathbf{A}_{b}$ for each test pixel is constructed using a dual concentric window, with an inner window region (IWR) centered within an outer window region (OWR), and only the pixels in the OWR will form the columns in $\mathbf{A}_{b}$ as shown in Figure 1(a). For example, if the size of OWR is $m \times m$ and the size of IWR is $t \times t$, where $t<m$, then the total number of pixels in the OWR that will form $\mathbf{A}_{b}$ is $m^{2}-t^{2}$, and hence, $\mathbf{A}_{b} \in \mathbb{R}^{p \times\left(m^{2}-t^{2}\right)}$.

Clearly, the dimension of IWR is very important and has strong effect on the detection performance since it is mainly used to enclose the targets of interests to be detected. It should be larger than or equal to the size of the desired targets of interests in the corresponding HSI, so as to exclude (but not completely) the probable target pixels from appearing in $\mathbf{A}_{b}$. It follows that, the main drawback of using an IWR is that one needs to know the upper bound of the target size and possibly its shape in order to choose a proper IWR. Unfortunately, this information is usually not at our disposal. This renders the choice of a proper IWR a difficult and tricky task, failing which, the detection performance can deteriorate significantly.

In this paper, we first develop the S-SRBBH model that is similar to SRBBH, but it further considers a simultaneous joint sparsity model [16] that incorporates the spatial correlation that exists within neighboring pixels in HSI. More precisely, for each test pixel, all its neighbors within a small neighborhood can be simultaneously rep- 
resented by a linear combination of a few common training samples but weighted with a different set of coefficients for each pixel. Next, the most challenging task in such a scheme is clearly that of accurately constructing $\mathbf{A}_{b}$ when the test pixel is a target. We want to achieve this task even without using an IWR in Figure 1(a). In fact, directly applying Figure 1(a) without an IWR to construct $\mathbf{A}_{b}$ will potentially deteriorates the detection performance. That is why, we propose two step-procedure that will be described in further details in subsection 2.2:

- Firstly, a low-rank and sparse matrix decomposition (LRaSMD) technique [17, 18] is applied to any given HSI to separate it into a low-rank background HSI and a sparse outlier HSI that contains the targets and possibly some additional noise errors.

- Secondly, for each test pixel, a concentric window is created on the low-rank background HSI, and each pixel in the window will contribute to one column in $\mathbf{A}_{b}$. Note that this concentric window does not require the information about the target size as most of the target pixels have been removed in the low-rank background HSI.

It should be remembered that the low-rank and sparse matrix decomposition does not always ensure the complete removal of the target corruption in the low-rank background HSI. This is due to the artifacts brought about by the optimization algorithms used in such LRaSMD techniques which typically involve convex relaxations or greedy techniques as surrogates.

The remainder of this paper is organized as follows. The SSRBBH model, as well as the procedure for constructing $\mathbf{A}_{b}$ using the LRaSMD technique, are briefly described in Section 2. The target detection capability of S-SRBBH when compared to some other detectors (SRBBH, AMF, ANMF and MSD) under different cases for constructing $\mathbf{A}_{b}$ is evaluated on the two real hyperspectral images presented in Section 3. Finally, Section 4 gives concluding remarks and some directions for future work.

\section{MAIN CONTRIBUTIONS}

\subsection{The Simultaneous SRBBH (S-SRBBH)}

It is expected that for real world HSI, neighboring pixels usually consist of similar materials and thus have similar spectral characteristics [16]. In this case, all the pixels in a small neighborhood can be simultaneously represented as a sparse linear combination of the common training samples but weighted with different coefficients. We define the matrix $\mathbf{X}=\left[\mathbf{x}_{1}, \mathbf{x}_{2}, \cdots, \mathbf{x}_{q}\right] \in \mathbb{R}^{p \times q}$, where $q$ is the total number of pixels in the neighborhood.

Note that if $q=1$, we return back to the SRBBH model. Hence, the S-SRBBH can be considered as a generalization of SRBBH when $q>1$. Therefore if $\mathbf{x} \in H_{0}$, we have:

$$
\begin{array}{cc}
\mathbf{x}_{1}= & c_{1,1} \mathbf{a}_{1}^{b}+c_{1,2} \mathbf{a}_{2}^{b}+\cdots+c_{1, N_{b}} \mathbf{a}_{N_{b}}^{b}, \\
\vdots & \\
\mathbf{x}_{q}= & c_{q, 1} \mathbf{a}_{1}^{b}+c_{q, 2} \mathbf{a}_{2}^{b}+\cdots+c_{q, N_{b}} \mathbf{a}_{N_{b}}^{b} .
\end{array}
$$

This implies that:

$$
\mathbf{X}=\left[\begin{array}{llll}
\mathbf{a}_{1}^{b} \mathbf{a}_{2}^{b} \cdots \mathbf{a}_{N_{b}}^{b}
\end{array}\right]\left[\mathbf{c}_{1} \mathbf{c}_{2} \cdots \mathbf{c}_{q}\right]=\mathbf{A}_{b} \mathbf{C}_{b},
$$

where $\mathbf{a}_{1}^{b}, \mathbf{a}_{2}^{b} \cdots, \mathbf{a}_{N_{b}}^{b}$ are the background training samples, $N_{b}$ is the total number of background training samples, $\mathbf{A}_{b} \in \mathbb{R}^{p \times N_{b}}$, and $\mathbf{C}_{b} \in \mathbb{R}^{N_{b} \times q}$.

Now, if $\mathbf{x} \in H_{1}$, we have:

$$
\begin{array}{r}
\mathbf{x}_{1}=c_{1,1}^{\prime} \mathbf{a}_{1}^{b}+c_{1,2}^{\prime} \mathbf{a}_{2}^{b}+\cdots+c_{1, N_{b}}^{\prime} \mathbf{a}_{N_{b}}^{b} \\
+z_{1,1} \mathbf{a}_{1}^{t}+z_{1,2} \mathbf{a}_{2}^{t}+\cdots+z_{1, N_{t}} \mathbf{a}_{N_{t}}^{t}, \\
\vdots \\
\mathbf{x}_{q}=c_{q, 1}^{\prime} \mathbf{a}_{1}^{b}+c_{q, 2}^{\prime} \mathbf{a}_{2}^{b}+\cdots+c_{q, N_{b}}^{\prime} \mathbf{a}_{N_{b}}^{b} \\
+z_{q, 1} \mathbf{a}_{1}^{t}+z_{q, 2} \mathbf{a}_{2}^{t}+\cdots+z_{q, N_{t}} \mathbf{a}_{N_{t}}^{t} .
\end{array}
$$

This implies that:

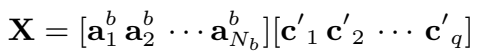

$$
\begin{aligned}
& +\left[\begin{array}{llll}
\mathbf{a}_{1}^{t} & \mathbf{a}_{2}^{t} & \cdots & \mathbf{a}_{N_{t}}^{t}
\end{array}\right]\left[\mathbf{z}_{1} \mathbf{z}_{2} \cdots \mathbf{z}_{q}\right] \\
& =\left[\begin{array}{ll}
\mathbf{A}_{b} & \mathbf{A}_{t}
\end{array}\right]\left(\begin{array}{l}
\mathbf{C}_{b}^{\prime} \\
\mathbf{z}_{t}
\end{array}\right)=\mathbf{A} \mathbf{S} .
\end{aligned}
$$

where $\mathbf{a}_{1}^{t}, \mathbf{a}_{2}^{t} \cdots, \mathbf{a}_{N_{t}}^{t}$ are the target training samples, $N_{t}$ denotes the total number of target training samples, $\mathbf{A}_{t} \in \Re^{p \times N_{t}}, \mathbf{A} \in$ $\mathbb{R}^{p \times\left(N_{b}+N_{t}\right)}$ is the union of $\mathbf{A}_{b}$ and $\mathbf{A}_{t}, \mathbf{C}_{b}^{\prime} \in \mathbb{R}^{N_{b} \times q}, \mathbf{Z}_{t} \in$ $\mathbb{R}^{N_{t} \times q}$, and $\mathbf{S} \in \mathbb{R}^{\left(N_{b}+N_{t}\right) \times q}$.

It is well known that the targets usually occupy a very small part of the whole HSI, and therefore, $\mathbf{N}_{t}<<\mathbf{N}_{b}$.

In fact, both $\mathbf{C}_{b}$ and $\mathbf{S}$ stand to be sparse, and therefore, the two (nonconvex and NP-HARD) minimization subproblems to solve are:

$$
\begin{gathered}
\hat{\mathbf{C}}_{b}=\underset{\mathbf{C}_{b}}{\operatorname{argmin}}\left\|\mathbf{A}_{b} \mathbf{C}_{b}-\mathbf{X}\right\|_{F} \text { s.t. }\left\|\mathbf{C}_{b}\right\|_{0,2} \leq K_{0}, \\
\hat{\mathbf{S}}=\underset{\mathbf{S}}{\operatorname{argmin}}\|\mathbf{A S}-\mathbf{X}\|_{F} \text { s.t. }\|\mathbf{S}\|_{0,2} \leq K_{0}^{\prime},
\end{gathered}
$$

where $\left\|\mathbf{C}_{b}\right\|_{0,2}$ and $\|\mathbf{S}\|_{0,2}$ denote the total number of nonzero rows of $\mathbf{C}_{b}$ and $\mathbf{S}$, respectively.

The method for constructing $\mathbf{A}_{b}$ based on the LRaSMD technique, as well as the construction of $\mathbf{A}_{t}$, are given in the next subsection.

In fact, $K_{0}$ and $K_{0}^{\prime}$ are a given upper bound on the sparsity level [19]. In fact, if $K_{0}$ and $K_{0}^{\prime}$ are set differently, this can lead to significantly weakened competition between $H_{0}$ and $H_{1}$. That is why, and to greatly reduce the complexity of the parameter adjustment, $K_{0}$ and $K_{0}^{\prime}$ are set equally to each other [15]. In this paper, we solve each subproblem using the Simultaneous Orthogonal Matching Pursuit (SOMP) [20] greedy algorithm. After that $\mathbf{C}_{b}$ and $\mathbf{S}$ are being estimated (to contain a few nonzero rows), the detection performance is evaluated as:

$$
D_{S-S R B B H}(\mathbf{x})=\left\|\mathbf{X}-\mathbf{A}_{b} \hat{\mathbf{C}}_{b}\right\|_{F}-\|\mathbf{X}-\mathbf{A} \hat{\mathbf{S}}\|_{F},
$$

where $\hat{\mathbf{S}}=\left(\begin{array}{c}\hat{\mathbf{C}}_{b}^{\prime} \\ \hat{\mathbf{z}}_{t}\end{array}\right)$. If $D_{S-S R B B H}(\mathbf{x})>\eta$ with $\eta$ being a prescribed threshold value, then $\mathbf{x}$ is declared as target; otherwise, $\mathbf{x}$ will be labeled as background.

\subsection{Construction of background and target dictionaries}

In fact, the targets in HSI always occupy a small part of the entire image and they are assumed to be randomly distributed and have low probability [21]. Hence, they are characterized by the sparsity property (in the spatial domain). The background is usually assumed to have a low-rank property. Recall that each pixel in HSI lies in a low dimensional subspace and thus can be represented by a few training samples. 


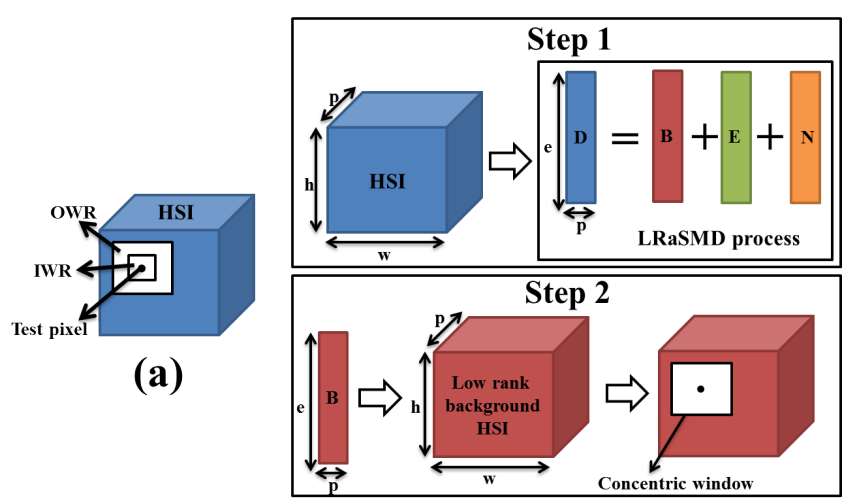

(b)

Fig. 1. (a) Dual concentric window for the $\mathbf{A}_{b}$ construction. (b) $\mathbf{A}_{b}$ construction based LRaSMD.

Based on the aforementioned analysis, the LRaSMD which form a matrix by adding an unknown sparse matrix to an unknown lowrank matrix is exploited to construct $\mathbf{A}_{b}$ as shown in both step 1 and step 2 of Figure 1(b). The construction of $\mathbf{A}_{b}$ via LRaSMD is described in the following two steps:

As a first step: for any HSI of size $h \times w \times p$, where $h$ and $w$ are the height and width of the image scene, respectively, and after rearranging it into a two-dimensional matrix $\mathbf{D}=\left[d_{i, j}\right] \in \mathbb{R}^{e \times p}$, where $e=h \times w$, the model of HSI can be modeled [21, 22] as $\mathbf{D}=\mathbf{B}+\mathbf{E}+\mathbf{N}$, where $\mathbf{B}=\left[b_{i, j}\right] \in \mathbb{R}^{e \times p}$ is the (low-rank) background matrix, $\mathbf{E}=\left[e_{i, j}\right] \in \mathbb{R}^{e \times p}$ is the (sparse) target matrix, and $\mathbf{N} \in \mathbb{R}^{e \times p}$ is usually assumed to be independent and identically distributed Gaussian noise.

As a second step: after that the background matrix $\mathbf{B}$ and the target matrix $\mathbf{E}$ are being estimated, we use only the estimation of $\mathbf{B}$ and we resize it to a cube of the same size $h \times w \times p$ (we shall call it as low-rank background HSI). Next, for each test pixel, we create a concentric window (on the low-rank background HSI), and all the pixels within the window will each contribute to one column in $\mathbf{A}_{b}$. Interestingly, one can expect to use the whole estimate of the matrix $\mathbf{B}$ and directly represent it as $\mathbf{A}_{b}$. In this case, the main drawback of this decision, regardless if a better construction of $\mathbf{A}_{b}$ can be obtained or not, is that the computational time of the algorithm will vastly increases, and which is not convenient for real world HSI.

Note that by resizing the estimate of $\mathbf{E}$ to a cube of size $h \times w \times p$, we shall call it as the sparse target HSI. In our paper, the sparse target HSI has no role since it is not used in neither constructing $\mathbf{A}_{b}$ nor $\mathbf{A}_{t}$. Since in hyperspectral target detection there is always a prior information about the target of interest to detect, the $\mathbf{A}_{t}$ is known and can be constructed using the MORTRAN atmosphericmodeling program [23] to generate a large number of target signatures under various atmospheric conditions. In addition, the $\mathbf{A}_{t}$ can also be formed by the USGS [24] and the ASTER [25] digital spectral libraries. In our paper, the $\mathbf{A}_{t}$ is simply formed from some of the target pixels present in the global image scene.

\subsection{Recovery of the low-rank and sparse components}

Recently, a number of LRaSMD optimization algorithms have been developed to recover both low-rank and sparse components. Among them, Candès et al. [17] proposed the robust principal component analysis (RPCA) which offers a blind separation of low-rank data and sparse noise. More precisely, the RPCA assumes that $\mathbf{D}=$ $\mathbf{B}+\mathbf{E}$ and therefore exactly decomposes $\mathbf{D}$ into $\mathbf{B}$ and $\mathbf{E}$. However, this exact decomposition does not always exist for real data matrix $\mathbf{D}$

That is why, Zhou and Tao [18] developed a more adaptive model, called the "Go decomposition" (GoDec). In GoDec, the exact RPCA decomposition of $\mathbf{D}$ does not exist due to an additive noise $\mathbf{N}$ and predefined $\operatorname{rank}(\mathbf{B}) \leq r, \operatorname{card}(\mathbf{E})<=k$, where $r$ and $k$ stand for the upper bound of the rank of $\mathbf{B}$ and the cardinality of $\mathbf{E}$, respectively. It assumes the following minimization problem:

$$
\underset{\mathbf{B}, \mathbf{E}}{\operatorname{argmin}}\|\mathbf{D}-\mathbf{B}-\mathbf{E}\|_{F}^{2} \text { s.t. } \operatorname{rank}(\mathbf{B}) \leq r, \operatorname{card}(\mathbf{E}) \leq k .
$$

Both $\mathbf{B}$ and $\mathbf{E}$ are recovered by alternatively solving the following minimization subproblems until convergence:

$$
\begin{gathered}
\mathbf{B}_{t}=\underset{\operatorname{rank}(\mathbf{B}) \leq r}{\operatorname{argmin}}\left\|\mathbf{D}-\mathbf{B}-\mathbf{E}_{t-1}\right\|_{F}^{2}, \\
\mathbf{E}_{t}=\underset{\operatorname{card}(\mathbf{E}) \leq k}{\operatorname{argmin}}\left\|\mathbf{D}-\mathbf{B}_{t}-\mathbf{E}\right\|_{F}^{2} .
\end{gathered}
$$

$\mathbf{B}_{t}$ can be updated via Bilateral Randomized Projections (BRP) based low-rank approximation [26]. More particularly, assume that $\mathbf{P}_{1}=\left(\mathbf{D}-\mathbf{E}_{t-1}\right) \mathbf{V}_{1}, \mathbf{V}_{2}=\mathbf{P}_{1}$, and $\mathbf{P}_{2}=\left(\mathbf{D}-\mathbf{E}_{t-1}\right)^{T} \mathbf{V}_{2}$, where $\mathbf{V}_{1} \in \mathbb{R}^{p \times r}$, and $\mathbf{V}_{2} \in \mathbb{R}^{e \times r}$ are two random matrices. The BRP-based rank $r$ approximation of $\mathbf{D} \in \mathbb{R}^{e \times p}$ is

$$
\mathbf{B}_{t}=\mathbf{P}_{1}\left(\mathbf{V}_{2}^{T} \mathbf{P}_{1}\right)^{-1} \mathbf{P}_{2}^{T} .
$$

The $\mathbf{E}_{t}$ in subproblem $6 \mathrm{~b}$ can be updated via entrywise hard thresholding of $\left(\mathbf{D}-\mathbf{B}_{t}\right)$.

In this paper, we exploit a variant of GoDec called "Semi-Soft GoDec" (SSGoDec) [27]. The SSGoDec is similar to GoDec, but it serves to adopt soft thresholding to the entries of $\mathbf{E}_{t}$ instead of hard thresholding. In this case, $\mathbf{E}$ is updated by:

$$
\mathbf{E}_{t}=\operatorname{sign}\left(\mathbf{D}-\mathbf{B}_{t}\right) \max \left(\left|\mathbf{D}-\mathbf{B}_{t}\right|-\lambda, 0\right),
$$

where $\lambda>0$ is a soft threshold. The main motivation of using SSGoDec instead of GoDec is that the former is 4 times faster than the latter while the error is kept the same or even smaller.

\section{SIMULATION RESULTS AND ANALYSIS}

The first HSI (DATA) [28] is an $201 \times 200$ image and consists of 167 spectral bands. We have only used a small zone (pixels in rows 1 to 150 and columns 80 to 180 ) for the detection. The main background materials of the selected zone are road and vegetation. There are three cars on the road and we will consider them as targets to be detected. Figure 2 exhibits the mean power in $\mathrm{dB}$ over the 167 bands.

The second HSI is the Pavia Center City (PaviaC). It is a 1096 $\times 1096$ image and consists of 102 bands in wavelengths ranging from 430 to $860 \mathrm{~nm}$. We used a small zone (pixels in rows 1 to 130 and columns 223 to 350 ) for the detection. The main background materials of this zone are bridge and water. There are some vehicles on the bridge and bare soil near the bridge pier and hence they will be selected as targets to be detected [22]. Figure 7 exhibits the mean power in $\mathrm{dB}$ over the 102 bands.

In the experiments, we considered three cases to form $\mathbf{A}_{b}$ :

- Case 1: using Figure 1(a) with IWR of size $5 \times 5$,

- Case 2: using Figure 1(a) but without IWR,

- Case 3: using Figure 1(b). 


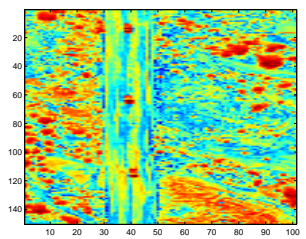

Fig. 2. DATA (average)
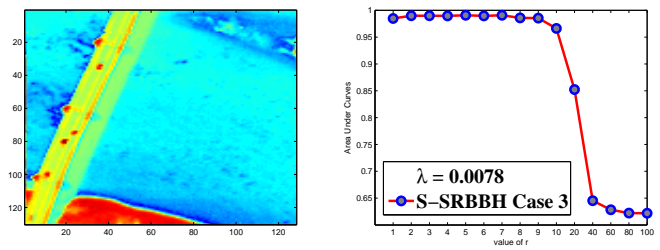

Fig. 7. PaviaC (average)

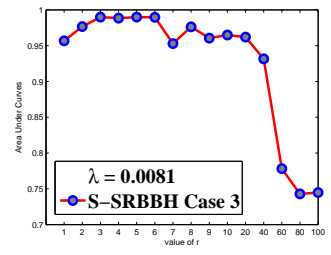

Fig. 3. AUCs: Case 3
Fig. 8. AUCs: Case 3

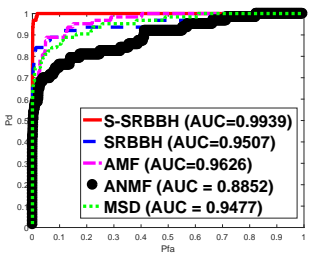

Fig. 4. ROC: Case 1

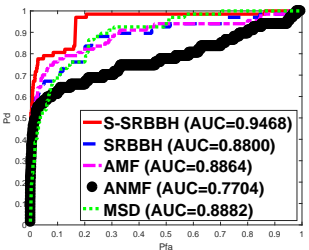

Fig. 9. ROC: Case 1

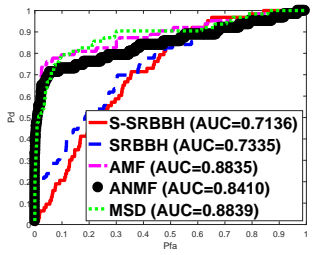

Fig. 5. ROC: Case 2

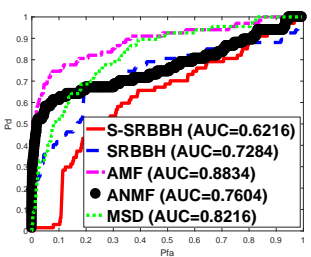

Fig. 10. ROC: Case 2

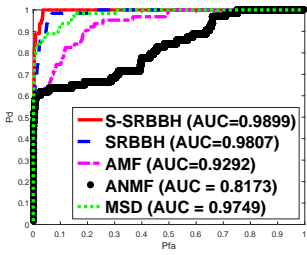

Fig. 6. ROC: Case 3

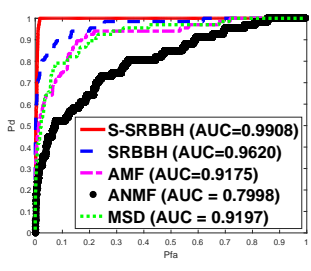

Fig. 11. ROC: Case 3
We evaluate the target detection performance of S-SRBBH by comparing it to some others (AMF, ANMF, MSD and SRBBH) on the different three cases. The detection performances are evaluated quantitatively by the receiver operating characteristics (ROC) curves which describe the probability of detection $\left(P_{d}\right)$ as a function of probability of false alarm $\left(P_{f a}\right)$. More particularly, we pick thousands of different thresholds $\eta$ between the minimal and maximal values of each detector output. The $P_{d}$ is determined as the ratio of the number of target pixels determined as target and the total number of true target pixels. Whereas the $P_{f a}$ can be calculated by the ratio of the number of false alarms (background pixels determined as targets) and the total number of pixels in the region test. In the implementation of AMF and ANMF, we used the robust Fixed Point (FP) [29] for covariance estimation. In addition, to obtain more stable inverse covariance, we regularized the FP as in [30] (shrinkage of the FP towards the identity matrix). In addition, the target signature we used for AMF and ANMF is the mean of target atoms $\mathbf{a}_{1}^{t}, \mathbf{a}_{2}^{t}, \cdots, \mathbf{a}_{N_{t}}^{t}$, where we choose $N_{t}=9$ for both DATA and PaviaC. In the case of MSD, the eigenvectors corresponding to the significant eigenvalues of the FP matrices obtained from $\mathbf{A}_{t}$ and $\mathbf{A}_{b}$ are used to generate the basis for target and background subspaces, respectively [31]. The OWR in Figure 1(a) is chosen to be of size $15 \times 15$ for all the detectors and we consider that the concentric window in Figure 1(b) is of the same size of OWR. We set $K_{0}=K_{0}^{\prime}=8$. We choose $q=25$, that is, a neighborhood of size $5 \times 5$. Importantly, the same Case (Case 1, 2 or 3 ) applied to construct $\mathbf{A}_{b}$ for S-SRBBH is also applied to all the other detectors in comparison. In addition, the choose of $\lambda$ is a hard problem and we found that the value $\lambda=1 / \sqrt{\max (e, p)}$ achieves good detection.

For DATA HSI: Figure 3 evaluates the S-SRBBH for Case 3 in terms of Area Under Curves (AUC) on different values of $r=1,2$, $3,4,5,6,7,8,9,10,20,40,60,80,100$. The highest AUC is 0.9899 at $r=3$, and hence, this value of $r$ will be used in Figure 6. Figure 4 evaluates the target detection performances of S-SRBBH when compared to other detectors for Case 1. Obviously, the S-SRBBH achieves the highest AUC ( $=0.9939)$. Both S-SRBBH and SRBBH $(A U C=0.9507)$ achieve good target detection since an IWR of the size of the desired targets is used in Figure 1(a). In Figure 5 (for Case 2), both S-SRBBH (AUC $=0.7136)$ and SRBBH (AUC $=0.7335)$ have the lowest AUC values comparing to the other detectors. This is to be expected since no IWR is used in Figure 1(a), and hence there are a lot corruption to $\mathbf{A}_{b}$ by the target pixels. Figure 6 shows that both S-SRBBH (AUC=0.9899) and SRBBH (AUC=0.9807) have the highest AUC values among the other detectors, as well as much higher AUC values than for Case 2 in Figure 5.

For PaviaC HSI: The same evaluations that have been made in Figure 3, 4, 5 and 6 are performed for PaviaC HSI in Figure 8, 9, 10 and 11, respectively. In Figure 8, the highest AUC is 0.9908 at $r=5$ and hence this value of $r$ will be used in Figure 11. Figure 9 (for Case 1) shows that $\mathrm{S}-\mathrm{SRBBH}$ achieves the highest AUC (=0.9468). Similarly, Figure 10 (for Case 2) demonstrates again that both S-SRBBH $(\mathrm{AUC}=6216)$ and SRBBH $(\mathrm{AUC}=7284)$ perform very poorly since no IWR is used in Figure 1(a). In Figure 11, we can notice again that both S-SRBBH (AUC $=0.9908)$ and SRBBH (AUC=0.9620) achieve the highest AUCs and much higher AUC values than for Case 2 in Figure 10. This demonstrates again that our use of LRaSMD based SSGoDec ensure a much less corruption to $\mathbf{A}_{b}$ by the target pixels than if Figure 1(a) is applied without an IWR.

\section{CONCLUSION}

In this paper, we first developed the S-SRBBH model that is similar to SRBBH but it further considers the interpixel correlation in hyperspectral imagery. Then, we served to achieve good target detection even without using an IWR in the $\mathbf{A}_{b}$ construction. This is done by first, exploiting the LRaSMD technique based on the SSGoDec optimization algorithm to approximately separate the given HSI into low-rank background HSI and sparse target HSI. Second, for each test pixel, a concentric window is used on the low-rank background HSI and all the pixels within the window are used to form $\mathbf{A}_{b}$. Two real hyperspectral images demonstrate that S-SRBBH has higher AUC values than of all the other detectors in comparison for both Case 1 and Case 3. In addition, exploiting the LRaSMD to construct $\mathbf{A}_{b}$ for both S-SRBBH and SRBBH greatly improves their target detection performances as shown between Case 2 and Case 3 .

As for future enhancements, our contribution would be to attempt to exploit our full knowledge about the target of interest to add constraints on the SSGoDec since its direct use does not ensure the complete removal of targets from the low-rank background HSI. 


\section{REFERENCES}

[1] G. Shaw and D. Manolakis, "Signal processing for hyperspectral image exploitation," IEEE Signal Processing Magazine, vol. 19, no. 1, pp. 12-16, Jan 2002.

[2] D. Manolakis, D. Marden, and G. Shaw, "Hyperspectral image processing for automatic target detection applications," Lincoln Laboratory Journal, vol. 14, no. 1, pp. 79-116, 2003.

[3] D. Manolakis, E. Truslow, M. Pieper, T. Cooley, and M. Brueggeman, "Detection algorithms in hyperspectral imaging systems: An overview of practical algorithms," IEEE Signal Processing Magazine, vol. 31, no. 1, pp. 24-33, Jan 2014.

[4] D. Manolakis, R. Lockwood, T. Cooley, and J. Jacobson, "Is there a best hyperspectral detection algorithm?," Proc. SPIE 7334, p. 733402, 2009.

[5] D. Manolakis and G. Shaw, "Detection algorithms for hyperspectral imaging applications," Signal Processing Magazine, IEEE, vol. 19, no. 1, pp. 29-43, 2002.

[6] J. Frontera-Pons, F. Pascal, and J. P. Ovarlez, "False-alarm regulation for target detection in hyperspectral imaging," in Computational Advances in Multi-Sensor Adaptive Processing (CAMSAP), 2013 IEEE 5th International Workshop on, Dec 2013, pp. 161-164.

[7] J. Frontera-Pons, M. A. Veganzones, S. Velasco-Forero, F. Pascal, J. P. Ovarlez, and J. Chanussot, "Robust anomaly detection in hyperspectral imaging," in 2014 IEEE Geoscience and Remote Sensing Symposium, July 2014, pp. 4604-4607.

[8] D. Manolakis, G. Shaw, and N. Keshava, "Comparative analysis of hyperspectral adaptive matched filter detectors," Proc. SPIE 4049, Algorithms for Multispectral, Hyperspectral, and Ultraspectral Imagery VI, vol. 2, Aug 2000.

[9] N. M. Nasrabadi, "Regularized spectral matched filter for target recognition in hyperspectral imagery," IEEE Signal Processing Letters, vol. 15, pp. 317-320, 2008.

[10] S. Kraut and L.L. Scharf, "The CFAR adaptive subspace detector is a scale-invariant GLRT," Signal Processing, IEEE Transactions on, vol. 47, no. 9, pp. 2538-2541, 1999.

[11] E. J. Kelly, "An adaptive detection algorithm," Aerospace and Electronic Systems, IEEE Transactions on, vol. 23, no. 1, pp. 115-127, November 1986.

[12] Scharf L. L. and B. Friedlander, "Matched subspace detectors," Signal Processing, IEEE Transactions on, vol. 42, no. 8, pp. 2146-2157, 1994.

[13] S. Kraut, L.L. Scharf, and L.T. Mc Whorter, "Adaptive Subspace Detector," Signal Processing, IEEE Transactions on, vol. 49, no. 1, pp. 1-16, Jan 2001.

[14] Y. Chen, N. M. Nasrabadi, and T. D. Tran, "Sparse representation for target detection in hyperspectral imagery," IEEE Journal of Selected Topics in Signal Processing, vol. 5, no. 3, pp. 629-640, June 2011.

[15] Y. Zhang, B. Du, and L. Zhang, "A sparse representation-based binary hypothesis model for target detection in hyperspectral images," IEEE Transactions on Geoscience and Remote Sensing, vol. 53, no. 3, pp. 1346-1354, March 2015.

[16] Y. Chen, N. M. Nasrabadi, and T. D. Tran, "Simultaneous joint sparsity model for target detection in hyperspectral imagery," IEEE Geoscience and Remote Sensing Letters, vol. 8, no. 4, pp. 676-680, July 2011.
[17] E. J. Candès, X. Li, Y. Ma, and J. Wright, "Robust principal component analysis?," J. ACM, vol. 58, no. 3, pp. 11:1-11:37, June 2011.

[18] T. Zhou and D. Tao, "Godec: Randomized low-rank \& sparse matrix decomposition in noisy case," in in International Conference on Machine Learning, 2011.

[19] J. A. Tropp and S. J. Wright, "Computational methods for sparse solution of linear inverse problems," Proceedings of the IEEE, vol. 98, no. 6, pp. 948-958, June 2010.

[20] J. A. Tropp, A. C. Gilbert, and M. J. Strauss Martin, "Algorithms for simultaneous sparse approximation: Part i: Greedy pursuit," Signal Process., vol. 86, no. 3, pp. 572-588, Mar. 2006.

[21] Shih-Yu Chen, Shiming Yang, Konstantinos Kalpakis, and Chein-I Chang, "Low-rank decomposition-based anomaly detection," 2013.

[22] Y. Zhang, B. Du, L. Zhang, and S. Wang, "A low-rank and sparse matrix decomposition-based mahalanobis distance method for hyperspectral anomaly detection," IEEE Transactions on Geoscience and Remote Sensing, vol. 54, no. 3, pp. 1376-1389, March 2016.

[23] A. Berk, L. Bernstein, and D. Robertson, "MORTRAN: A moderate resolution model for LOWTRAN 7," Tech. Rep. GLTR-90-0122, Geophysics Laboratory, Bedford, MA, 1989.

[24] Roger N. Clark, Gregg A. Swayze, Andrea J. Gallagher, Trude V.V. King, and Wendy M. Calvin, "The U. S. Geological Survey, Digital Spectral Library: Version 1: 0.2 to 3.0 micros," Open file report, U.S. Geological Survey, 1993.

[25] A.M. Baldridge, S.J. Hook, C.I. Grove, and G. Rivera, "The ASTER Spectral Library Version 2.0," Remote Sensing of Environment, vol. 113, pp. 711-715, 2009.

[26] T. Zhou and D. Tao, "Bilateral random projections," in Information Theory Proceedings (ISIT), 2012 IEEE International Symposium on, July 2012, pp. 1286-1290.

[27] T. Zhou and D. Tao, "Shifted subspaces tracking on sparse outlier for motion segmentation," in Proceedings of the TwentyThird International Joint Conference on Artificial Intelligence. 2013, IJCAI '13, pp. 1946-1952, AAAI Press.

[28] E. Terreaux, J. P. Ovarlez, and F. Pascal, "Anomaly detection and estimation in hyperspectral imaging using random matrix theory tools," in Computational Advances in Multi-Sensor Adaptive Processing (CAMSAP), 2015 IEEE 6th International Workshop on, Dec 2015, pp. 169-172.

[29] D. E. Tyler, "A distribution-free m-estimator of multivariate scatter," The Annals of Statistics, vol. 15, no. 1, pp. 234-251, Mar 1987.

[30] Y. Chen, A. Wiesel, and A. O. Hero, "Robust shrinkage estimation of high-dimensional covariance matrices," Signal Processing, IEEE Transactions on, vol. 59, no. 9, pp. 4097-4107, 2011.

[31] H. Kwon and N. M. Nasrabadi, "A comparative analysis of kernel subspace target detectors for hyperspectral imagery," EURASIP Journal on Advances in Signal Processing, 2006. 